\title{
Toughness, Tenacity and Maximum Initial Strength of Rubber Modified Asphalt Binders
}

\author{
Kazem Jadidi \\ Howard R. Hughes College of \\ Engineering, University of \\ Nevada, Las Vegas, USA \\ kazem.jadidi@unlv.edu
}

\author{
Mehdi Khalili \\ Howard R. Hughes College of \\ Engineering, University of \\ Nevada, Las Vegas, USA \\ mehdi.khalili@unlv.edu
}

\author{
Moses Karakouzian \\ Howard R. Hughes College of \\ Engineering, University of \\ Nevada, Las Vegas, USA \\ moses.karakouzian@unlv.edu
}

\author{
Serji Amirkhanian \\ College of Engineering, \\ University of Alabama, \\ Tuscaloosa, Alabama, USA \\ samirkhanian@eng.ua.edu
}

\begin{abstract}
The toughness and tenacity test method, which was developed in the $1980 \mathrm{~s}$, is popular for evaluating a polymermodified binder. Several states like Nevada require performing this test to evaluate non-modified binder samples, as well as other types of modified binders. In this regard, a toughness and tenacity test was performed on rubber-modified samples produced from virgin binder PG58-28, PG64-16 and AC-20. In order to take the rubber size, type and content into account, two rubber sizes, mesh \#20 and \#40, two rubber types, ambient and cryogenic, and three rubber contents, $10 \%, 15 \%$, and $20 \%$ were produced and tested. The results then were compared with polymer-modified and terminally blended rubber-modified samples. The results show improvement in the amount of initial maximum strength, and a decline in the magnitude of elongation, toughness and tenacity for the rubber-modified binder, compared to other types of binders.
\end{abstract}

Keywords-rubber binder; ambient; cryogenic; toughness; tenacity

\section{INTRODUCTION}

The toughness and tenacity is a test designed to discover the elastomeric properties of asphalt. Asphalt's ability to be stretched is measured and presented by these two parameters [1]. In addition, for the analysis of the results of this experiment, a third parameter called maximum initial strength is measured. Toughness of the asphalt binder is the area underneath the curve of variation of force versus elongation, and represents the strength of the asphalt binder as well as the capability to be stretched. Tenacity is the area underneath the curve of variation of force versus elongation after the initial strength has been overcome, and represents the capability of the asphalt binder to be stretched after the initial strength has been overcome [2, 4]. This method of testing, which is based on an experiment presented by Benson in 1955 for rubberized asphalts, was developed for polymer modified asphalt in the 1980s [3].

Toughness is equal to the total work that is required to separate the testing equipment ball from binder [5]. For bituminous material, high toughness will lead to more flexibility and consequently, better performance against repeated loadings. Moreover, toughness is lower in cold temperatures in comparison to higher temperatures [6]. Asphalt binders with higher strength and toughness demonstrate better resistance against surface abrasion [7]. Toughness and tenacity reflects the adhesion properties of an asphalt binder [8]. It is possible to determine the tensile strength of an asphalt binder based on the results of a toughness and tenacity test [9]. Sulphur improves the toughness and tenacity of polymer modified asphalt [10]. This test is among the quality assurance tests required for most state transportation departments [11]. Asphalt binders with higher toughness and tenacity will demonstrate greater resistance against deformation in warm weather. Moreover, aging will lead to a decline in toughness and tenacity properties of asphalt [12]. For a styrenebutadiene-styrene (SBS) modified binder, the highest toughness and tenacity happens with $6 \%$ SBS concentration [13], while it will decline with an SBS concentration over 7\% [14]. Increasing styrene copolymer content from 3 to 6 in a polymer modified binder improves toughness and tenacity of the original binder significantly [15]. Other investigations demonstrate that styrene-butadiene rubber (SBR) modified asphalt also presents compatible toughness and tenacity values in comparison with other types of binders [16]. It has been discovered that an increase in rubber content leads to a decrease in toughness properties of rubberized asphalt concrete [17]. Warm mix asphalt has lower toughness value compared to non-modified asphalt [18]. On the other hand, although CRM additives diminish the modified asphalt stiffness at lower temperatures, they increase the toughness of rubber modified asphalt [19]. Toughness has been investigated more in places with low temperature [20].

\section{METHODOLGY}

In order to produce rubber modified asphalt, virgin asphalt bitumen was obtained from local manufacturing companies and mixed with crumb rubber. Virgin binders were used to produce rubber modified asphalt. Three types of virgin binders used in manufacturing rubber modified asphalt were PG 64-16, PG 58-28, AC-20. In order to perform a better comparison, polymer modified asphalt as well as terminal blended rubberize asphalt also were provided. For this reason, polymer modified PG 64-28 NV and PG 76-22 NV, as well as terminally blend rubber modified asphalt PG64-22TR were prepared. From each sample, three specimens were tested, and at the end, the average values were used in the analysis. 


\section{A. Sample Preparation}

Three CRM contents, $10 \%, 15 \%$, and $20 \%$, were mixed with each virgin binder. In order to take into account the influence of rubber particle size and gradation in modified binders, two maximum sizes, mesh \#20 and mesh \#40, were selected for CRM particles. Both ambient and cryogenic CRMs were used. Moreover, in order to modify asphalt binders, both cryogenic and ambient methods were used to produce crumb rubber. Two main methods for grinding crumb rubber include the ambient and cryogenic methods. In the ambient method, the rubber particle distributions expand between $75 \mu \mathrm{m}$ and $5 \mathrm{~mm}$. Rubber particles manufactured in this method have a rough texture which leads to higher surface area. This is because in the ambient method, rubber specimens are scraped in a tearing process. In contrast, in the cryogenic method, liquid nitrogen is used to freeze scrap tire, and then the frozen tire rubbers are crushed into particles between 025 inches and mesh \#30 with a hammer [21]. Figure 1 demonstrates the combination of original, rubber modified, and polymer modified asphalt samples, which were used in performing this research.

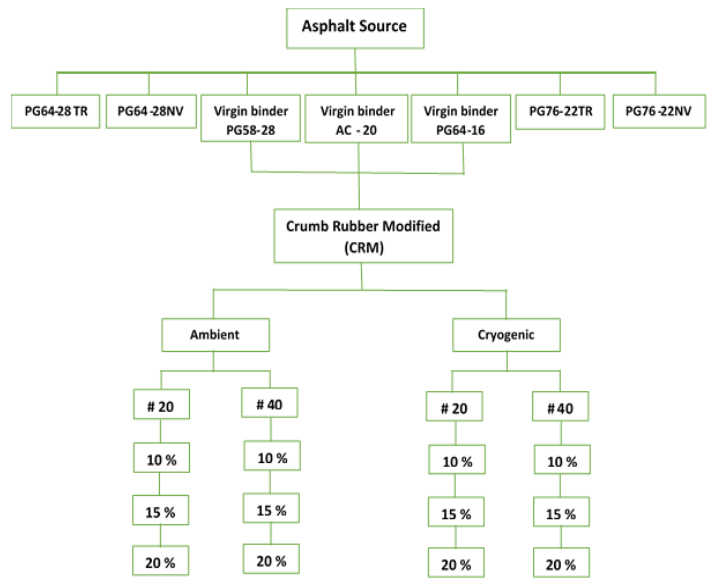

Fig. 1. Asphalt source combination

\section{B. Testing of the Original and Modified Samples}

Toughness and tenacity tests were performed on rubbermodified asphalt binders, as well as polymer-modified asphalt and terminally blended rubber modified binders. The results are presented in graphs and tables for better comparison. In order to compare the rubber-modified binders with other traditional specimens, both rubber size and content played a prominent role in evaluating the properties of rubber-modified binders. Figure 2 illustrates a schematic of the toughness and tenacity test.

\section{RESULTS AND DISCUSSION}

Because the results of the experiments are to be presented to the Nevada Department of Transportation (DOT), the toughness and tenacity tests were conducted in accordance with Nev. 745I [22] and ASTM D5801. Although both standards present recommendations for polymer modified asphalt and in general this type of testing is more popular for polymer modified binders, the same procedure was used in conducting this research to conform with Nevada DOT requirements. All tests were performed at $25^{\circ} \mathrm{C}$ in accordance with the standard.

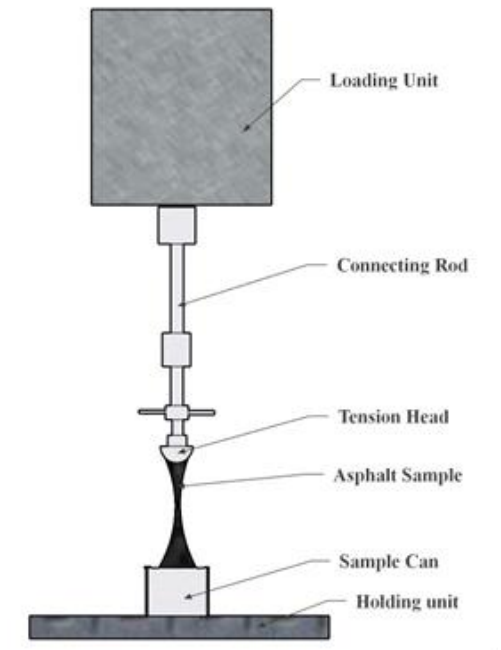

Fig. 2. Schematic of toughness and tenacity test

\section{A. Force anb Elongation}

The virgin binder samples were modified with two rubber types and two rubber sizes, so the results will be presented in four sets of graphs. These sets of graphs include ambient (Am) and cryogenic $(\mathrm{Cr})$ for rubber types, and \#40 and \#20 for rubber particle sizes. The results of the force and elongation for rubber modified binders made with virgin asphalt PG58-28 are presented in Figure 3. In comparison with rubber modified binder PG 58-28, for modified binder AC-20, elongation forces do not follow a remarkable pattern for various rubber sizes and types, except for ambient \#20. Generally, there is a considerable increase in the amount force in which each sample failed. Modified binder with $15 \%$ rubber ambient \#20 presents the highest resistance force, which is about 150lbs. All binder samples were able to resist against more than 100lbs of stretching force.

The results of the experiment for samples manufactured with binder PG64-16 is similar to AC-20, but with several differences. It can be seen from the graphs presented in Figures 3-4 that $10 \%$ rubber leads to lower stretching forces while samples modified with $20 \%$ rubber demonstrate higher resistance to stretching before failure. On the other hand, adding more rubber improves the initial strength against stretching for modified binders. In terms of elongation, all samples presented lower magnitude. It is obvious from Figures $3-4$, that regardless of binder type and rubber particle size and type, the rate of stretching is less than 1.5 inches, which is not considerable. On the other hand, while rubber improves the initial strength of the binder, it decreases the elongation rate. However, polymer modified and terminally blended rubber modified binder samples demonstrated noticeably higher values of elongation, ranging between 4 and 18 inches. In contrast, the magnitude of force was slightly lower for these specimens in comparison to rubber modified binders. Toughness, tenacity and maximum initial strength were calculated based on elongation vs. force graphs which were presented in Figures 3-4. In order to make a better comparison, toughness, tenacity and maximum initial strength were calculated for a polymer modified binder and a terminally 
blended rubber modified binder. The results are presented below. Toughness (TS), tenacity (TY), maximum initial strength (MIS), and elongation for non-modified PG58-28 and PG64-16, as well as polymer modified and terminally blended rubber modified samples are presented in Table I. Based on Table I, non-modified binder samples present the lowest value for toughness, tenacity and maximum initial strength. For these samples, the amount of elongation is slightly higher than for rubber-modified binders, but still lower than polymer modified and terminally blended rubber modified specimens. In general, polymer modified and terminally blended rubber modified samples present the highest values, specifically in terms of toughness, tenacity, and elongation.

(a)
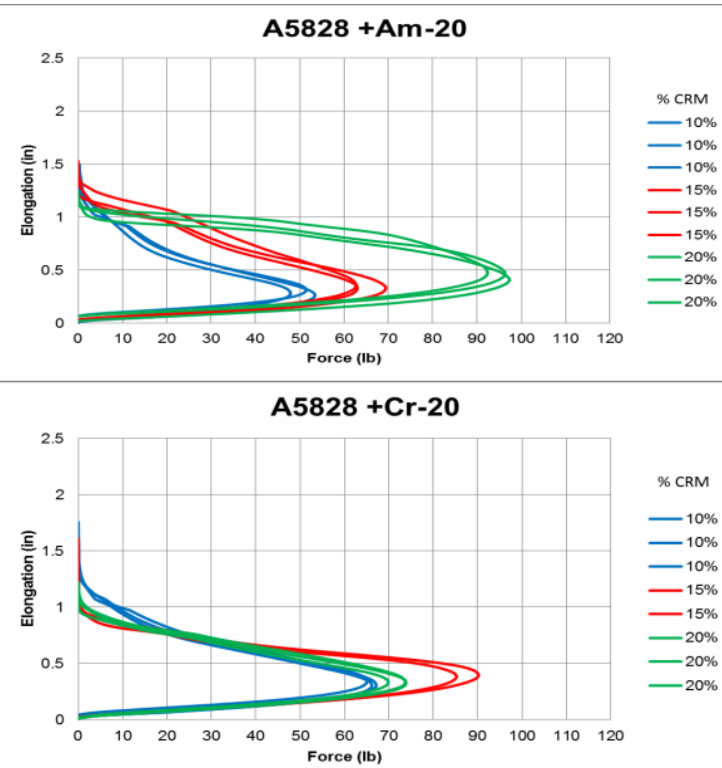

$\%$ CRM

(b)

(c)
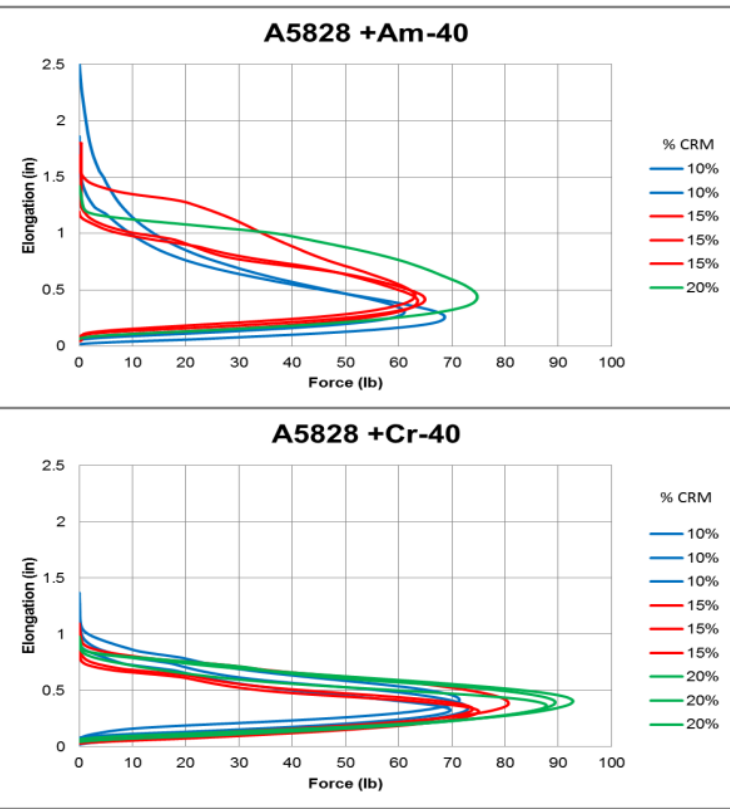

Fig. 3. Force vs. elongation for binder AC - 20 mixed with rubber: (a) ambient \#20, (b) cryogenic \#20, (c) ambient \#40 and (d) cryogenic \#40 (a)
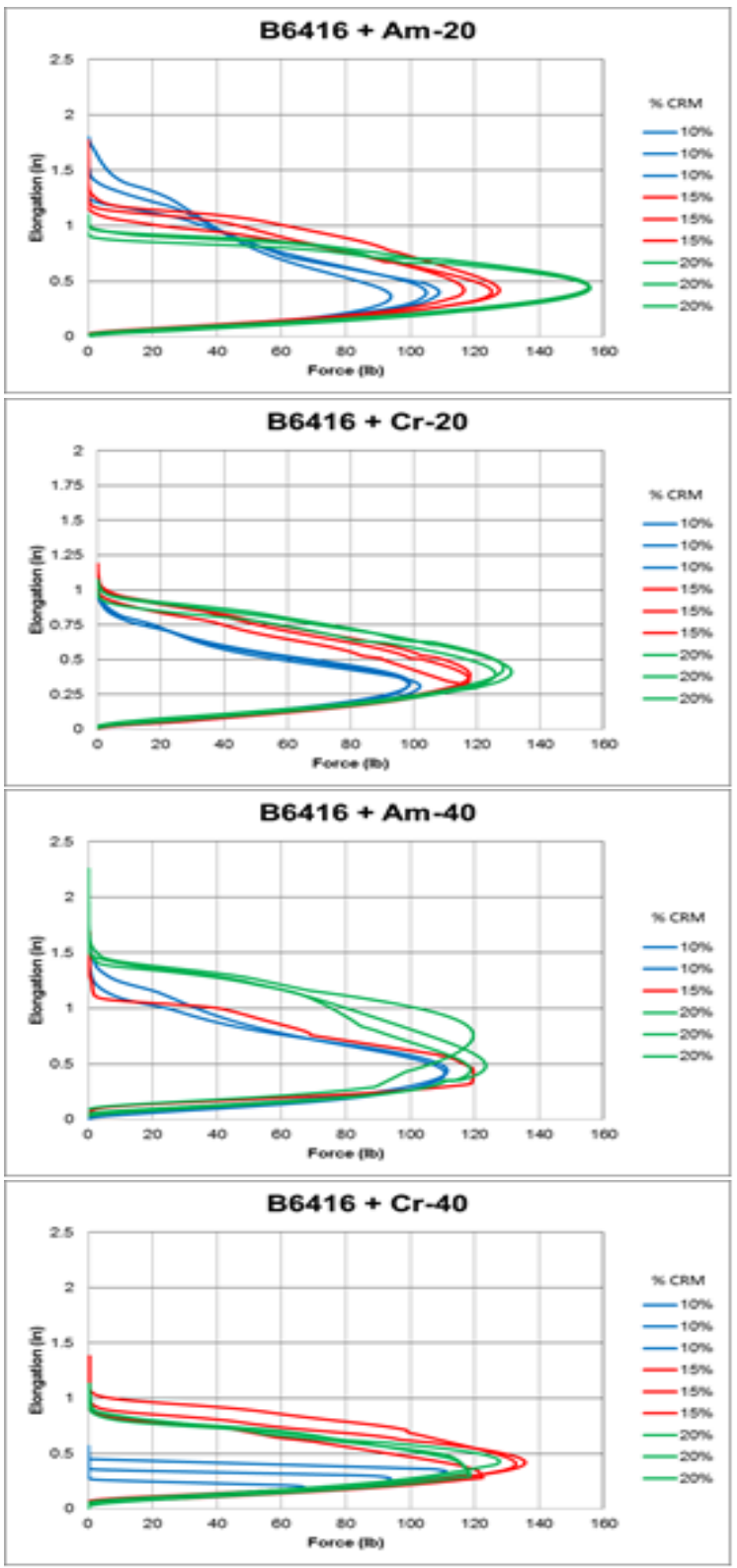

Fig. 4. Force vs. elongation for binder PG64-16 mixed with rubber: (a) ambient \#20, (b) cryogenic \#20, (c) ambient \#40 and (d) cryogenic \#40

TABLE I. TEST RESULTS

\begin{tabular}{|c|c|c|c|c|}
\hline Sample & $\begin{array}{c}\text { TS } \\
\text { (in-lb) }\end{array}$ & $\begin{array}{c}\text { TY } \\
\text { (in-lb) }\end{array}$ & $\begin{array}{c}\text { MIS } \\
\text { (lb) }\end{array}$ & Elongation (in) \\
\hline PG58-28 & 15 & 3 & 26.3 & 2.5 \\
\hline PG64-16 & 46 & 6 & 77.3 & 3.5 \\
\hline PG64-28 NV & 510 & 458 & 82.2 & 14 \\
\hline PG64-28 TR & 365 & 316 & 77 & 18 \\
\hline PG76-22 NV & 111 & 80 & 27.8 & 5 \\
\hline PG76-22 TR & 126 & 88 & 35.8 & 4 \\
\hline
\end{tabular}

\section{B. Toughness, Tenacity and Maximum Initial Strength}

The calculated toughness values for rubber modified samples, polymer modified samples, and terminally blended rubber modified samples are presented in Figures 5-7. Considering the fact that this test originally was developed for 
polymer modified binder, in this experiment also, toughness and tenacity test was performed on polymer modified binder and the results were used as a base for comparison. In addition, the results are compared with the toughness and maximum initial strength of terminally blended specimens.

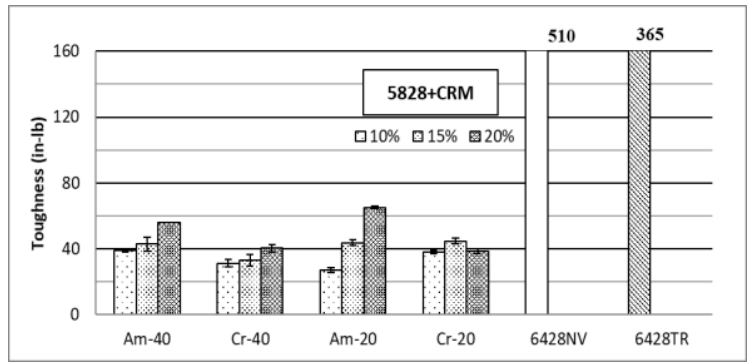

Fig. 5. Toughness, rubber-modified binder PG (58-28)

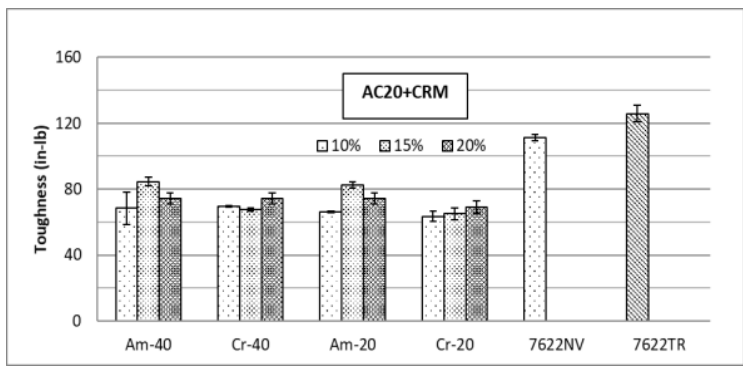

Fig. 6. Toughness, rubber-modified binder ac-20

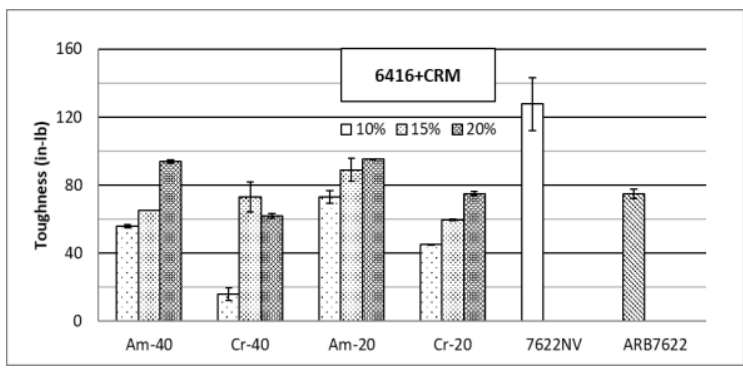

Fig. 7. Toughness, rubber-modified binder PG (64-16)

Regarding Figures 5-7, there is considerable difference in sample toughness between rubber modified binders and base specimens. Binder PG64-28 presented higher toughness values compared to other binder sources, with polymer modified samples showing the highest values and terminally blended rubber modified of the same binder source in second place. Polymer modified and terminally blended rubber modified binder PG76-22 lead to higher values in comparison to rubber modified samples, although the difference is not considerable compared to some rubber modified specimens like PG64-16 20\% AM \#40 and PG64-16 20\% AM \#20. Among rubber modified specimens, the highest values, with toughness magnitude slightly over 90 in-lb, belong to PG64-16 modified with 20 percent ambient rubber. Rubber modified PG64-16 and AC-20 presented higher values compared to PG58-28. Moreover, no remarkable difference was observed between ambient and cryogenic rubber types or for various rubber gradations. In general, higher rubber content leads to greater toughness values which means rubber improves the toughness properties of modified binders. The tenacity values for rubber modified binders for all samples were considerably low. They were not comparable with base samples, so the graphs for tenacity results are not presented in this paper. In contrast, rubber modified specimens demonstrated higher maximum initial strength (MIS) values. The results are illustrated in Figures 8-10.

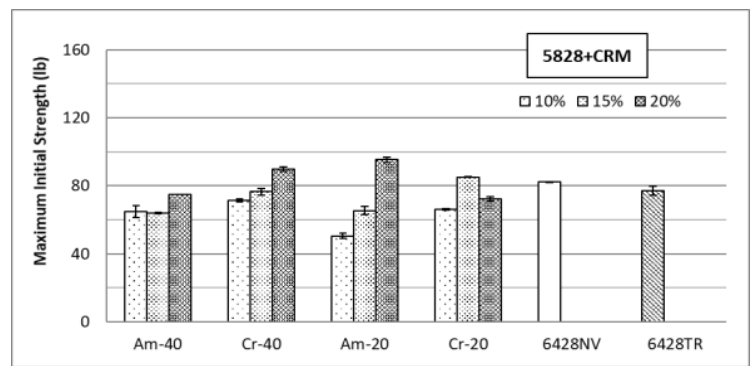

Fig. 8. MIS of CRM binder PG (58-28) and base samples

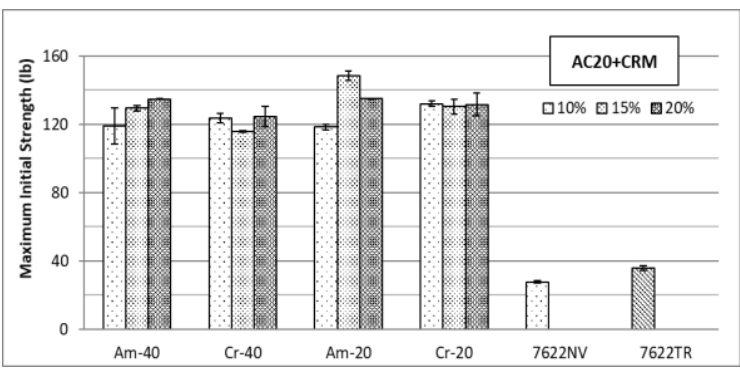

Fig. 9. MIS of CRM binder ac-20 and base samples

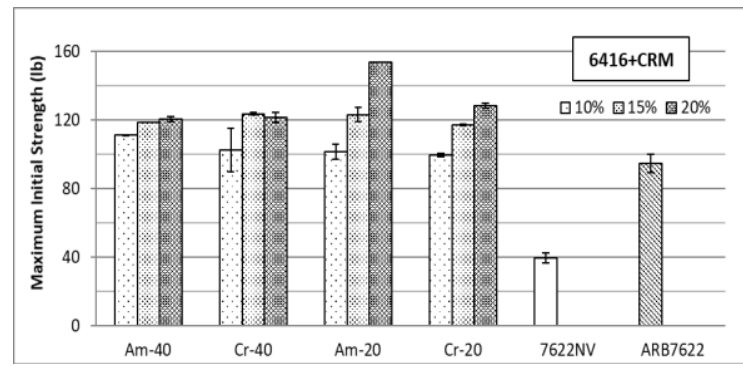

Fig. 10. MIS of CRM binder PG (64-16) and base samples

Rubber modified binders presented higher maximum initial strength values in comparison with polymer modified and terminally blended rubber modified binder specimens. Similar to toughness, higher initial strength belongs to modified specimens manufactured with binder AC-20 and PG 64-16, while modified PG28-28 presented lower values. For most samples, a direct correlation between rubber content and initial strength was observed, which means that higher rubber content leads to enhancement in the initial strength of a modified binder. For each binder source, there was no indication that the rubber type and size had any influence. The lowest maximum initial strength values belong to polymer modified and terminally blended rubber modified PG76-22 specimens. 


\section{SUMMARY AND CONCLUSIONS}

Toughness and tenacity test method was used to evaluate rubber modified binder samples. The results were compared with polymer modified and terminally blended rubber modified binder specimens. Consideration was taken to discover the influences of rubber size, type, and content. Toughness, tenacity, and maximum initial strength of samples were calculated and compared. The results of this investigation are summarized as follows:

- Overall results indicated that the CRM additives improve the maximum initial strength of virgin binders.

- The amount of toughness, tenacity, and elongation declined remarkably for rubber modified specimens, in comparison with polymer modified and terminally blended samples.

- For rubber modified binders, PG64-22 modified with 20\% rubber ambient \#20 presented the highest toughness value.

- Among rubber modified specimens, 20\% ambient \#20 rubber modified PG64-16 demonstrated the highest maximum initial strength.

- Increase of rubber particle content led to improvement of the toughness properties of modified samples, while rubber size and type did not show significant influence.

\section{REFERENCES}

[1] ASTM D5801-17, Standard Test Method for Toughness and Tenacity of Asphalt Materials, ASTM Book of Standards, ASTM, 2017

[2] O. E. Brisco, Asphalt Rheology: Relationship to Mixture, ASTM Special Technical Publication STP 941-EB. Michigan, ASTM, 1987

[3] J. Read, D. Whiteoak, The Shel Bitumen Handbook, Thomas Telford 2003

[4] J. S. Chen, K. Y. Lin, "Mechanism and behavior of bitumen strength reinforcement using fibers", Journal of Materials Science, Vol. 40, No. 1, pp. 87-95, 2005

[5] J. S. Chen, M. C. Liao, H. H. Tsai, "Evaluation and optimization of the engineering properties of polymer-modified asphalt", Practical Failure Analysis, Vol. 2, No. 3, pp. 75-83, 2002

[6] A. Modarres, "Investigating the toughness and fatigue behavior of conventional and SBS modified asphalt mixes", Construction and Building Materials, Vol. 47, pp. 218-222, 2013

[7] J. S. Clites, H. A. Colvin, D. F. Klemmensen, Polymer for Asphalt Cement Modification, U.S. Patent No. 5,986,010, 1999

[8] P. Liang, M. Liang, W. Fang, Y. Zhang, C. Qian, S. Ren, "Improving thermo-rheological behavior and compatibility of SBR modified asphalt by addition of polyphosphoric acid (PPA)", Construction and Building Materials, Vol. 139, pp. 183-192, 2017

[9] H. Zhou, S. E. Nodes, J. E. Nichols, "Evaluation of Three Polymer Modified Asphalt Concretes", Transportation Research Record, Vol. 1454, pp. 181-192, 1994

[10] F. Zhang, J. Yu, "The research for high-performance SBR compound modified asphalt", Construction and Building Materials, Vol. 24, No. 3, pp. $410-418,2010$

[11] Z. Hossain, D. Ghosh, M. Zaman, K. Hobson, "Use of the multiple stress creep recovery (MSCR) test method to characterize polymer-modified asphalt binders", Journal of Testing and Evaluation, Vol. 44, No. 1, pp. 507-520, 2015

[12] Z. Jiang, C. Hu, S. M. Easa, X. Zheng, Y. Zhang, "Evaluation of physical, rheological, and structural properties of vulcanized EVA/SBS modified bitumen", Journal of Applied Polymer Science, Vol. 134, No. 21,2017
[13] J. S. Chen, M. C. Liao, C. H. Lin, "Determination of polymer content in modified bitumen", Materials and Structures, Vol. 36, No. 9, pp. 594598, 2003

[14] H. Akiyoshi, S. Ueno, A. Kasahara, K. Saito, "Effect of the morphology of SBS modified asphalt on mechanical properties of binder and mixture", Journal of the Eastern Asia Society for Transportation Studies, Vol. 6, pp. 1153-1167, 2005

[15] L. H. Lewandowski, D. F. Klemmensen, SBR for Asphalt Cement Modification, U.S. Patent No. 6,136,899, 2000

[16] T. S. Shuler, J. H. Collins, J. P. Kirkpatrick, "Polymer-modified asphalt properties related to asphalt concrete performance", in: Asphalt Rheology: Relationship to Mixture, ASTM, 1987

[17] F. Xia, S. N. Amirkhanian, "Laboratory investigation of moisture damage-e in rubberised asphalt mixtures containing reclaimed asphalt pavement”, International Journal of Pavement Engineering, Vol. 10, No. 5, pp. 319-328, 2009

[18] F. Xiao, W. Zhao, T. Gandhi, S. Amirkhanian, "Influence of antistripping additives on moisture susceptibility of warm mix asphalt mixtures", Journal of Materials in Civil Engineering, Vol. 22, No. 10, pp. 1047-1055, 2010

[19] H. Wang, Z. You, J. M. Beale, P. Hao, "Laboratory evaluation on high temperature viscosity and low temperature stiffness of asphalt binder with high percent scrap tire rubber", Construction and Building Materials, Vol. 26, No. 1, pp. 583-590, 2012

[20] K. W. Kim, S. J. Kweon, Y. S. Doh, T. S. Park, "Fracture toughness of polymer-modified asphalt concrete at low temperatures", Canadian Journal of Civil Engineering, Vol. 30, No. 2, pp. 406-413, 2003

[21] M. H. Blumenthal, "Producing ground scrap tire rubber: a comparison between ambient and cryogenic technologies", in: Proceedings of the 17th Biennial Waste Processing Conference, ASME, 1996

[22] State of Nevada, Department of Transportation, Method of Test for Toughness and Tenacity of Polymer Modified Asphalt, Test Method Nev. T745I, State of Nevada, Department of Transportation, Materials Division 2011 